\title{
ON $n$-MAXIMAL SUBALGEBRAS OF LIE ALGEBRAS
}

\author{
DAVID A. TOWERS \\ Department of Mathematics, Lancaster University \\ Lancaster LA1 4YF, England \\ d.towers@lancaster.ac.uk
}

\begin{abstract}
A chain $S_{0}<S_{1}<\ldots<S_{n}=L$ is a maximal chain if each $S_{i}$ is a maximal subalgebra of $S_{i+1}$. The subalgebra $S_{0}$ in such a series is called an $n$-maximal subalgebra. There are many interesting results concerning the question of what certain intrinsic properties of the maximal subalgebras of a Lie algebra $L$ imply about the structure of $L$ itself. Here we consider whether similar results can be obtained by imposing conditions on the $n$-maximal subalgebras of $L$, where $n>1$.
\end{abstract}

\section{Introduction}

Throughout $L$ will denote a finite-dimensional Lie algebra over a field $F$. A chain $S_{0}<S_{1}<\ldots<S_{n}=L$ is a maximal chain if each $S_{i}$ is a maximal subalgebra of $S_{i+1}$. The subalgebra $S_{0}$ in such a series is called an $n$-maximal subalgebra. There are many interesting results concerning the question of what certain intrinsic properties of the maximal subalgebras of a Lie algebra $L$ imply about the structure of $L$ itself. For example: all maximal subalgebras are ideals of $L$ if and only if $L$ is nilpotent (see [1]); all maximal subalgebras of $L$ are c-ideals of $L$ if and only if $L$ is solvable (see [13]); if $L$ is solvable then all maximal subalgebras have codimension one in $L$ if and only if $L$ is supersolvable (see [2]); $L$ can be characterised when its maximal subalgebras satisfy certain lattice-theoretic conditions, such as 
modularity (see [15]). Our purpose here is to consider whether similar results can be obtained by imposing conditions on the $n$-maximal subalgebras of $L$, where $n>1$.

Similar studies have proved fruitful in group theory (see, for example, [4], [5] and [7]). For Lie algebras the following result was established by Stitzinger.

Theorem 1.1 (Stitzinger, [9, Theorem]) Every 2-maximal subalgebra of L is an ideal of $L$ if and only if one of the following holds:

(i) $L$ is nilpotent and $\phi(L)=\phi(M)$ for all maximal subalgebras $M$ of $L$;

(ii) $\operatorname{dim} L=2$; or

(iii) $L$ is simple and every proper subalgebra is one-dimensional.

In the above result $\phi(L)$ denotes the Frattini ideal of $L$; that is, the largest ideal contained in the intersection of the maximal subalgebras of $L$. Our first objective in the next section is to find a similar characterisation of Lie algebras in which all 2-maximal subalgebras are subideals, and then those in which they are nilpotent. In section three we consider when all 3-maximals are ideals, and when they are subideals. In the final section we look at the situation where every $n$-maximal subalgebra is a subideal.

\section{2-maximal subalgebras}

First, the following observations will be useful.

Lemma 2.1 Let $A / B$ be a chief factor of $L$ with $A \subseteq \phi(L)$. Then $A / B$ is an irreducible $L / \phi(L)$-module.

Proof. The nilradical, $N$, of $L$ is the intersection of the centralizers of the factors in a chief series of $L$, by [3, Lemma 4.3]. Since $\phi(L) \subseteq N$ this implies that $[A, \phi(L)] \subseteq B$ and so the multiplication of $L$ on $A$ induces a module action of $L / \phi(L)$ on $A / B$. Hence $A / B$ can be viewed as an irreducible $L / \phi(L)$-module.

We will refer to a chief factor such as is described in Lemma 2.1 as being below $\phi(L)$. 
Lemma 2.2 If every $n$-maximal subalgebra of $L$ is a subideal of $L$, then every $(n-1)$-maximal subalgebra is nilpotent.

Proof. Let $J$ be an $(n-1)$-maximal subalgebra of $L$. Then every maximal subalgebra $I$ of $J$ is an $n$-maximal subalgebra of $L$ and so is a subideal of $L$, and thus of $J$. It follows that $I$ is an ideal of $J$, and hence that $J$ is nilpotent, by [1].

Theorem 2.3 Every 2-maximal subalgebra of $L$ is a subideal of $L$ if and only if one of the following holds:

(i) L is nilpotent;

(ii) $L=N+F x$ where $N$ is the nilradical, $N^{2}=0$ and adx acts irreducibly on $N$; or

(iii) $L$ is simple with every proper subalgebra one dimensional.

Proof. Let every 2-maximal of $L$ be a subideal of $L$. If $L$ is simple then (iii) holds with $\phi(L)=0$. So suppose that $N$ is a maximal ideal of $L$. Since $N$ will be contained in a maximal subalgebra of $L$ it will be nilpotent, by Lemma 2.2.

Suppose first that $N \nsubseteq \phi(L)$. Then there is a maximal subalgebra $M$ of $L$ such that $L=N+M$. Since $M$ is nilpotent, $L$ is solvable. Moreover, $L$ is nilpotent or minimal non-nilpotent. Suppose that $L$ is minimal nonnilpotent and $\phi(L) \neq 0$, so $L=N+F x$ where $N$ is the nilradical of $L$, $N^{2}=\phi(L)$ and ad $x$ acts irreducibly on $N / N^{2}$, by [12, Theorem 2.1]. But now $\phi(L)+F x$ is a maximal subalgebra of $L$ and any 2-maximal subalgebra of $L$ containing $F x$ would have to be contained in a proper ideal of $L$, which would be nilpotent, by Lemma 2.2, and so contained in $N$. It follows that $\phi(L)=0$. Hence either (i) or (ii) holds.

So suppose now that $N \subseteq \phi(L)$. Then $N=\phi(L)$ and $L / \phi(L)$ is simple with every proper subalgebra one dimensional. Now $N+F s$ is a maximal subalgebra of $L$ for every $s \in S$, and, as in the preceding paragraph, any 2-maximal subalgebra containing $F s$ would be contained in $N$. It follows that $N=0$.

Conversely, let $L$ satisfy (i), (ii) or (iii). If $L$ is nilpotent then every subalgebra of $L$ is a subideal of $L$. If (ii) holds then the maximal subalgebras of $L$ are $N$ and $F x$, and so the 2-maximal subalgebras are inside $N$ and so are subideals of $L$. If (iii) holds then the only 2-maximal subalgebra is the trivial subalgebra. 
Note that, over a perfect field $F$ of characteristic zero or $p>3$, for $L$ to satisfy condition (iii) in Theorem 1.1, it must be three-dimensional and $\sqrt{F} \nsubseteq F$, by [14, Theorem 3.4].

Next we consider when all of the 2-maximal subalgebras are nilpotent. We consider the non-solvable and solvable cases separately, as for the former case we require restrictions on the field $F$.

Theorem 2.4 Let $L$ be a non-solvable Lie algebra over an algebraically closed field $F$ of characteristic different from 2,3. Then every 2-maximal subalgebra of $L$ is nilpotent if and only if $L / \phi(L) \cong \operatorname{sl}(2)$ and $\operatorname{sl}(2)$ acts nilpotently on $\phi(L)$. If $F$ has characteristic zero or if $L$ is restricted, then $\phi(L)=0$.

Proof. Suppose that every 2-maximal subalgebra of $L$ is nilpotent, and let $M$ be a maximal subalgebra of $L$. If $M$ is not nilpotent then there is an element $x \in M$ such that ad $\left.x\right|_{M}$ has a non-zero eigenvalue, $\lambda$ say. But now $M=F x+F y$ since this is not nilpotent. Hence, every maximal subalgebra of $L$ is nilpotent or two dimensional; in particular, they are all solvable. If $F$ has characteristic $p>3$, it follows from [17, Proposition 2.1] that $L / \phi(L) \cong \operatorname{sl}(2)$. Moreover, all maximal subalgebras of $\operatorname{sl}(2)$ are two dimensional, so $\phi(L)+F x$ is nilpotent for every $x \in \operatorname{sl}(2)$. The claim for characteristic zero is well known; that for the case when $L$ is restricted is [17, Corollary 2.13]

The converse is easy.

Theorem 2.5 Let $L$ be a solvable Lie algebra over a field $F$. Denote the image of a subalgebra $S$ of $L$ under the canonical homomorphism onto $L / \phi(L)$ by $\bar{S}$. Then all 2-maximal subalgebras of $L$ are nilpotent if and only if one of the following occurs:

(i) L is nilpotent;

(ii) L is minimal non-nilpotent, and so is as described in [12];

(iii) $\bar{L}=\bar{A} \dot{+} F \bar{b}$, where $\bar{A}$ is the unique minimal abelian ideal of $\bar{L}$ and $\phi(L)+F b$ is minimal non-nilpotent;

(iv) $\bar{L}=\bar{A} \dot{+}\left(F \overline{b_{1}}+F \overline{b_{2}}\right)$, where $\bar{A}$ is a minimal abelian ideal of $\bar{L}, \bar{B}=$ $F \overline{b_{1}}+F \overline{b_{2}}$ is a subalgebra of $\bar{L}$ and $L / \phi(L)$ acts nilpotently on $\phi(L)$; or

(v) $\bar{L}=\left(\overline{A_{1}} \oplus \overline{A_{2}}\right) \dot{+} F \bar{b}$, where $\overline{A_{1}}$ and $\overline{A_{2}}$ are minimal abelian ideals of $\bar{L}$ and $L / \phi(L)$ acts nilpotently on $\phi(L)$. 
Proof. Suppose that all 2-maximal subalgebras of $L$ are nilpotent. Then $\bar{L}=\left(\bar{A}_{1} \oplus \ldots \oplus \bar{A}_{n}\right) \dot{+} \bar{B}$, where $\bar{A}_{i}$ is a minimal abelian ideal of $\bar{L}$ for each $i=1, \ldots, n, \overline{A_{1}} \oplus \ldots \oplus \overline{A_{n}}$ is the nilradical, $\bar{N}$, of $\bar{L}$ and $\bar{B}$ is a subalgebra of $\bar{L}$, by [11, Theorem 7.3]. If $n>2$ we have $\bar{A}_{i}+\bar{B}$ is nilpotent for each $i=1, \ldots, n$. But then $\bar{L}$, and hence $L$, is nilpotent, by [11, Theorem 6.1]. Suppose that $\operatorname{dim} \bar{B}>2$ and let $\bar{C}$ be a minimal ideal of $\bar{B}$. If $\operatorname{dim} \bar{C}=1$ then $\bar{N}+\bar{C}$ is a nilpotent ideal of $\bar{L}$, contradicting the fact that $\bar{N}$ is the nilradical of $\bar{L}$; if $\operatorname{dim} \bar{C}>1$ we have that $\bar{N}+F \bar{c}$ is nilpotent for each $\bar{c} \in \bar{C}$ which again implies that $\bar{N}+\bar{C}$ is a nilpotent ideal of $\bar{L}$. Finally, if $n=2$ and $\operatorname{dim} \bar{B}=2$ a similar argument produces a contradiction.

So suppose next that $n=1$ and $\operatorname{dim} \bar{B}=1$. Then the maximal subalgebras of $L$ are $A$ and $\phi(L)+F x$, where $x \notin N$. If $\phi(L)+F b$ is nilpotent we have case (ii); if it is minimal non-nilpotent we have case (iii).

Next let $n=1$ and $\operatorname{dim} \bar{B}=2$. If $B=F b_{1}+F b_{2}$ then $A+F b_{1}$ and $A+F b_{2}$ are maximal subalgebras of $L$, and so $\phi(L)+F b_{1}$ and $\phi(L)+F b_{2}$ are 2-maximal subalgebras. It follows that $B$ acts nilpotently on $\phi(L)$ and we have case (iv).

Finally, suppose that $n=2$ and $\operatorname{dim} \bar{B}=1$. Maximal subalgebras are $A_{1} \oplus A_{2}, A_{1}+F b$ and $A_{2}+F b$, and $\phi(L)+F b$ is a 2-maximal subalgebra. It follows that $F b$ acts nilpotently on $\phi(L)$ and we have case (v).

The converse is straightforward.

If $S$ is a subalgebra of $L$, the centraliser of $S$ in $L$ is $C_{L}(S)=\{x \in$ $L:[x, S]=0\}$.

Corollary 2.6 With the notation of Theorem [2.5, if $L$ is solvable and $F$ is algebraically closed, then all 2-maximal subalgebras of $L$ are nilpotent if and only if one of the following occurs:

(a) L is nilpotent;

(b) $\operatorname{dim} L \leq 3$;

(c) F has characteristic $p, \bar{L}=\oplus_{i=0}^{p-1} F \overline{a_{i}}+F \overline{b_{1}}+F \overline{b_{2}}$, where $\left[\overline{a_{i}}, \overline{b_{1}}\right]=a_{i+1}$, $\left[\overline{a_{i}}, \overline{b_{2}}\right]=(\alpha+i) \overline{a_{i}}$ for $i=0, \ldots, p-1$ ( $\alpha \in F$, suffices modulo $\left.p\right)$, $\left[\overline{b_{1}}, \overline{b_{2}}\right]=\overline{b_{1}}$ and $L / \phi(L)$ acts nilpotently on $\phi(L)$; or

(d) $\bar{L}=F \overline{a_{1}}+F \overline{a_{2}}+F \bar{b}$, where $\left[\bar{b}, \overline{a_{1}}\right]=\overline{a_{1}},\left[\bar{b}, \overline{a_{2}}\right]=\alpha \overline{a_{2}}(\alpha \in F)$, $\left[\overline{a_{1}}, \overline{a_{2}}\right]=0$ and $L / \phi(L)$ acts nilpotently on $\phi(L)$.

Proof. We consider in turn each of the cases given in Theorem 2.5, Clearly case (i) gives (a), and if case (ii) holds then $\operatorname{dim} L=2$ (see [12]), which is 
included in (b). If case (iii) holds, then $\bar{A}$ and $\phi(L)$ are both one dimensional, and so we have (b) again.

Consider next case (iv). Suppose first that $\bar{B}$ is abelian. Then $\operatorname{dim} \bar{A}=1$, by [10, Lemma 5.6]. But now $\operatorname{dim} \bar{L} / C_{\bar{L}}(F \bar{a}) \leq 1$ so $\operatorname{dim} C_{\bar{L}}(F \bar{a}) \geq 2$, contradicting the fact that $C_{\bar{L}}(F \bar{a})=F \bar{a}$. Thus $B$ cannot be abelian.

If $\bar{B}$ is non-abelian, then $\bar{B}=F \overline{b_{1}}+F \overline{b_{2}}$ where $\left[\overline{b_{1}}, \overline{b_{2}}\right]=\overline{b_{1}}$. If $F$ has characteristic zero, then $\operatorname{dim} \bar{A}=1$, by Lie's Theorem. But now, as in the previous paragraph, $\operatorname{dim} C_{\bar{L}}(F \bar{a}) \geq 2$, yielding the same contradiction. Hence $F$ has characteristic $p>0$. Then this algebra has a unique $p$-map

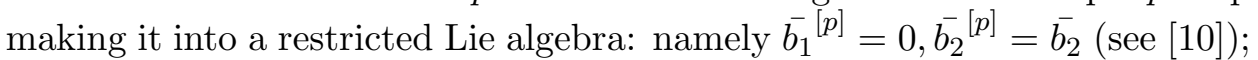
its irreducible modules are of dimension one or $p$, by [10, Example 1, page 244]. Once again we can rule out the possibility that $\operatorname{dim} \bar{A}=1$. So suppose now that $\operatorname{dim} \bar{A}=p$. Let $\alpha$ be an eigenvalue for ad $\left.\bar{b}_{2}\right|_{\bar{A}}$, so $\left[\bar{a}, \overline{b_{2}}\right]=\alpha \bar{a}$ for some $\bar{a} \in \bar{A}$. Then $\left[\bar{a}\left(\operatorname{ad} \overline{b_{1}}\right)^{i}, \overline{b_{2}}\right]=(\alpha+i) \bar{a}\left(\operatorname{ad} \overline{b_{1}}\right)^{i}$ for every $i$, so putting $\overline{a_{i}}=\bar{a}\left(\operatorname{ad} \overline{b_{1}}\right)^{i}$ we see that $F \overline{a_{0}}+\cdots+F a_{p-1}^{-}$is $\bar{B}$-stable and hence equal to $\bar{A}$. We then have the multiplication given in (e).

Finally, consider case (v). Then $\overline{A_{1}}$ and $\overline{A_{2}}$ are one-dimensional. Moreover, if $L$ is not nilpotent, the $\bar{b}$ must act non-trivially on at least one of them, $\overline{A_{1}}=F \overline{a_{1}}$, say. This gives the multiplication described in (f).

\section{3-maximals subalgebras}

We consider first Lie algebras all of whose 3-maximal subalgebras are ideals. We shall need the following lemma, which is an easy generalisation of 9 , Lemma 2].

Lemma 3.1 Suppose that every $n$-maximal subalgebra of $L$ is an ideal of $L$. Then every $(n-1)$-maximal subalgebra of $L$ is nilpotent and is either an ideal or is one dimensional.

Proof. Let $K$ be a $(n-1)$-maximal subalgebra of $L$. The fact that $K$ is nilpotent follows from Lemma 2.2. Suppose that $\operatorname{dim} K>1$. Then $K$ has at least two distinct maximal subalgebras $J_{1}$ and $J_{2}$, by [9. Lemma 1]. These are $n$-maximal subalgebras of $L$ and so are ideals of $L$. Moreover, $K=J_{1}+J_{2}$ and so is an ideal of $L$.

Theorem 3.2 Let $L$ be a solvable Lie algebra over a field $F$. Then every 3-maximal subalgebra of $L$ is an ideal of $L$ if and only if one of the following holds: 
(i) $L$ is nilpotent and $\phi(K)=\phi(M)$ for every 2-maximal subalgebra $K$ of $L$ and every maximal subalgebra $M$ of $L$ containing it; or

(ii) $\operatorname{dim} L \leq 3$.

Proof. Suppose that every 3-maximal subalgebra of $L$ is an ideal of $L$. Then Lemma 3.1 shows that $L$ is given by Theorem 2.5. We consider each of the cases in turn, and use the notation of that result. Suppose first that $L$ is nilpotent and let $J$ be a 3-maximal subalgebra of $L, K$ be any 2maximal subalgebra of $L$ containing it, and $M$ be any maximal subalgebra of $L$ containing $K$. Then $J$ is an ideal of $L$ and $M / J$ is two dimensional. It follows that $M^{2} \subseteq J$ and so $M^{2} \subseteq \phi(K)=K^{2} \subseteq M^{2}$. Hence $\phi(K)=M^{2}=$ $\phi(M)$.

Now suppose that $\bar{L}=\bar{A} \dot{+} \bar{B}$, where $\bar{A}$ is the unique minimal ideal of $\bar{L}$ and $\bar{B}$ is a subalgebra of $\bar{L}$ with $\operatorname{dim} \bar{B} \leq 2$. This covers cases (ii), (iii) and (iv) of Theorem 2.5. If $\operatorname{dim} \bar{A}>2$ then there is a proper subalgebra $\bar{C}$ of $\bar{A}$ which is a 3 -maximal subalgebra of $\bar{L}$, and so an ideal of $\bar{L}$, contradicting the minimality of $\bar{A}$. If $\operatorname{dim} \bar{B}=2$ then $A+F b$ is a maximal subalgebra of $L$ for each $\overline{0} \neq \bar{b} \in \bar{B}$. It follows that $\phi(L)+F b$ is a 2 -maximal subalgebra of $L$. If this is an ideal of $L$ then $F \bar{b}$ is a minimal ideal of $\bar{L}$, contradicting the uniqueness of $\bar{A}$. It follows from Lemma 3.1 that it has dimension one, and so $\phi(L)=0$. Similarly, $\operatorname{dim} \bar{A}=2$ yields that $\phi(L)=0$. Hence $\phi(L) \neq 0$ implies that $\operatorname{dim}(L / \phi(L) \leq 2$ and thus that $L$ is nilpotent. So suppose that $\phi(L)=0$ and $\operatorname{dim} L=4$. Then $A+F b$ is a maximal subalgebra for every $b \in B$, and so $F a$ is a 3 -maximal subalgebra, and hence an ideal, of $L$ for every $a \in A$, contradicting the minimality of $A$. Thus $\operatorname{dim} L \leq 3$.

So, finally, suppose that case (v) of Theorem 2.5 holds. We have that $\phi(L)=0$ as in the paragraph above. Also, if $\operatorname{dim} A_{i}>1(i=1,2)$ there is a proper subalgebra $C$ of $A_{i}$ which is a 3 -maximal subalgebra, and hence an ideal, of $L$. It follows that $\operatorname{dim} A_{i}=1$ for $i=1,2$ and $\operatorname{dim} L=3$.

Conversely, suppose that (i) or (ii) hold. If (ii) holds then every 3maximal is 0 and thus an ideal of $L$, so suppose that (i) holds. Let $J$ be a 3-maximal subalgebra of $L$. Then $J$ is a maximal subalgebra of a 2-maximal subalgebra $K$ of $L$ and $M^{2}=\phi(M)=\phi(K) \subseteq J$ for every maximal subalgebra $M$ containing $K$. It follows that $J$ is an ideal of $M$. But now $\operatorname{dim} L / J=3$ and there are two maximal subalgebras $M_{1}$ and $M_{2}$ of $L$ containing $J$ with $L=M_{1}+M_{2}$. Since $J$ is an ideal of $M_{1}$ and $M_{2}$, it is an ideal of $L$.

Theorem 3.3 Let $L$ be a non-solvable Lie algebra over a field $F$. Then every 3-maximal subalgebra of $L$ is an ideal of $L$ if and only if one of the 
following holds:

(i) $L$ is simple, all 2-maximal subalgebras of $L$ are at most one dimensional and at least one of them has dimension one;

(ii) $L / Z(L)$ is a simple algebra, all of whose maximal subalgebras are one dimensional, $Z(L)=\phi(L)$ and $\operatorname{dim} Z(L)=0$ or 1 ;

(iii) $L=S \dot{+} F x$ where $S$ is a simple ideal of $L$ and all maximal subalgebras of $S$ are one dimensional.

Proof. Suppose that every 3-maximal subalgebra of $L$ is an ideal of $L$. Clearly, if $L$ is simple then every 2-maximal subalgebra has dimension at most one, by Lemma 3.1, and so satisfies (i) or (ii). So let $N$ be a maximal ideal of $L$. Suppose first that $\operatorname{dim} L / N=1$, so $L=N \dot{+} F x$, say. Clearly $N$ has more than one maximal subalgebra, since otherwise it is one dimensional and $L$ is solvable. If $N$ has a maximal subalgebra $K_{1}$ that is an ideal of $L$, then $N=K_{1}+K_{2}$, where $K_{2}$ is another maximal subalgebra of $L$, and both $K_{1}$ and $K_{2}$ are nilpotent. But then $N$, and hence $L$, is solvable. It follows from Lemma 3.1 that every maximal subalgebra of $N$ is one dimensional. Let $I$ be a non-trivial ideal of $N$. Then $\operatorname{dim} N / C_{N}(I) \leq 1$. But this implies that $\operatorname{dim} N=2$ and $L$ is solvable again. It follows that $N$ is simple with all maximal subalgebras one dimensional. Hence, $L$ is as in case (iii).

So suppose now that $L / N$ is simple. Then all 2-maximal subalgebras of $L / N$ have dimension at most one. Suppose first that $L / N$ has a onedimensional 2-maximal subalgebra $A / N$. Then $\operatorname{dim} A=1$, by Lemma 3.1, and so $N=0$ and we have case (i) again. So suppose now that all maximal subalgebras of $L / N$ are one dimensional. Then $N$ is nilpotent and if $K$ has codimension one in $N, K$ is an ideal of $L$. Moreover, $K+F s$ is a 2 maximal subalgebra of $L$ for every $s \notin N$. It follows from Lemma 3.1 that $K=0$. Hence $\operatorname{dim} N=1$. But now $\operatorname{dim} L / C_{L}(N) \leq 1$, which implies that $N=Z(L)$. If $Z(L)=\phi(L)$ we have case (ii). If $Z(L) \neq \phi(L)$ then we have a special case of (iii).

The converse is straightforward.

Corollary 3.4 Let $L$ be a non-solvable Lie algebra over an algebraically closed field $F$ of characteristic different from 2,3. Then every 3-maximal subalgebra of $L$ is an ideal of $L$ if and only if $L \cong \operatorname{sl}(2)$.

Proof. Suppose that every 3-maximal subalgebra of $L$ is an ideal of $L$. Then every 2-maximal subalgebra of $L$ is nilpotent, so $L / \phi(L) \cong s l(2)$, by Theorem 2.4, But $\phi(L)=0$ by Theorem 3.3. The converse is clear. 
Next we give a characterisation of those Lie algebras in which every 3 -maximal subalgebra is a subideal.

Theorem 3.5 Let $L$ be a solvable Lie algebra over a field $F$. Then every 3maximal subalgebra of $L$ is a subideal of $L$ if and only if one of the following occurs:

(i) $L$ is nilpotent;

(ii) $L=N \dot{+} F b$ where $N$ is the nilradical, $\operatorname{dim} N^{2}=1$, adb acts irreducibly on $N / N^{2}$, and $N^{2}+F b$ is abelian;

(iii) $\bar{L}=\bar{A} \dot{+} F \bar{b}$, where $\bar{A}$ is the unique minimal abelian ideal of $\bar{L}, \phi(L)^{2}=$ 0 and $\phi(L)$ is an irreducible Fb-module;

(iv) $L=A \dot{+}\left(F b_{1}+F b_{2}\right)$, where $A$ is a minimal abelian ideal of $L$, and $B=F b_{1}+F b_{2}$ is a subalgebra of $L$; or

(v) $L=\left(A_{1} \oplus A_{2}\right) \dot{+} F b$, where $A_{1}$ and $A_{2}$ are minimal abelian ideals of $L$.

Proof. Suppose that every 3-maximal subalgebra of $L$ is a subideal of $L$. Then $L$ is as described in Theorem 2.5. We consider each of the cases in turn. In case (i) every subalgebra of $L$ is a subideal of $L$. Suppose that case (ii) holds, so $L=N \dot{+} F b$ where $N / N^{2}$ is a faithful irreducible $F b$-module and $N^{2}+F b$ is nilpotent. Let $C$ be an ideal of $N^{2}+F b$ of codimension one in $N^{2}$. Then $C+F b$ is a 2-maximal subalgebra of $L$. Suppose that $C \neq 0$. Then $b \in D$ where $D \subset C+F b$ is a 3 -maximal subalgebra of $L$. Since $D$ is a nilpotent subideal of $L$, there is a $k \in \mathbb{N}$ such that $N(\operatorname{ad} D)^{k}=0$. Since $b \in D$ and $N / N^{2}$ is faithful, this is impossible. Hence $D=0$ and $\operatorname{dim} N^{2}=1$.

Suppose that (iii) holds. Then $\phi(L) / \phi(L)^{2}$ is a faithful irreducible $F b$ module, and so $\phi(L)^{2}+F b$ is a 2-maximal subalgebra of $L$. If $\phi(L)^{2} \neq 0$ then $b \in D$ where $D \subset \phi(L)^{2}+F b$ is a 3-maximal subalgebra of $L$. But this yields a contradiction as in the preceding paragraph.

Suppose next that (iv) holds. Then we can choose $b_{1}, b_{2}$ so that $\left[\overline{b_{1}}, \overline{b_{2}}\right]=$ $\lambda \overline{b_{2}}$ where $\lambda=0,1$. Then $\left[\bar{A}, \overline{b_{2}}\right]$ is an ideal of $\bar{L}$ and so is equal to $\bar{A}$, since, otherwise, $\overline{b_{2}} \in C_{\bar{L}}(\bar{A})=\bar{A}$. Now $\phi(L)+F b_{2}$ is a 2-maximal subalgebra of $L$. Thus, if $\phi(L) \neq 0$ we have that $b_{2}$ belongs to a 3-maximal subalgebra of $L$, giving a contradiction again.

Finally, suppose that (v) holds. Then $\phi(L)+F b$ is a 2-maximal subalgebra of $L$ and we conclude that $\phi(L)=0$ as above.

Conversely, if any of these cases are satisfied then every 3-maximal subalgebra of $L$ is inside the nilradical of $L$, and hence is a subideal of $L$. 
Proposition 3.6 Let $L$ be a non-solvable Lie algebra over an algebraically closed field $F$ of characteristic different from 2,3. Then every 3-maximal subalgebra of $L$ is a subideal of $L$ if and only if $L / \phi(L) \cong \operatorname{sl}(2)$.

Proof. Suppose that every 3-maximal subalgebra of $L$ is a subideal of $L$. Then every 2-maximal subalgebra of $L$ is nilpotent, so $L / \phi(L) \cong s l(2)$, by Theorem 2.4. Conversely, if $L / \phi(L) \cong s l(2)$ then every 3-maximal subalgebra of $L$ is contained in $\phi(L)$, which is nilpotent, and so they are all subideals of $L$.

\section{4 n-maximal subalgebras}

The following result was proved by Schenkman in [8] for fields of characteristic zero, and can be extended to cover a large number of cases in characteristic $p$ by using a result of Maksimenko from [6].

Lemma 4.1 Let I be a nilpotent subideal of a Lie algebra $L$ over a field $F$. If $F$ has characteristic zero, or has characteristic $p$ and $L$ has no subideal with nilpotency class greater than or equal to $p-1$, then $I \subseteq N$, where $N$ is the nilradical of $L$.

Proof. If $F$ has characteristic zero this is [8, Lemma 4]. For the characteristic $p$ case we follow Schenkman's proof. Let $I$ be a nilpotent subideal of $L$ and suppose that $I=I_{0}<I_{1}<\ldots<I_{n}=L$ is a chain of subalgebras of $L$ with $I_{j}$ an ideal of $I_{j+1}$ for $j=0, \ldots, n-1$. Let $N_{j}$ be the nilradical of $I_{j}$ and let $x_{j} \in I_{j}$. Then $I \subseteq N_{1}$, since $I$ is a nilpotent ideal of $I_{1}$. Also $\left[I_{j}, x_{j+1}\right] \subseteq I_{j}$, and so ad $x_{j+1}$ defines a derivation of $I_{j}$ for each $j=0, \ldots, n-1$. Moreover, $N_{j}$ is a subideal of $L$ and so has nilpotency class less than $p-1$. It follows from [6, Corollary 1] that $\left[N_{j}, x_{j+1}\right] \subseteq N_{j}$, and hence that $N_{j}$ is an ideal of $I_{j+1}$. But then $N_{j} \subseteq N_{j+1}$, and $I \subseteq N_{1} \subseteq N_{2} \subseteq \ldots \subseteq N_{n}=N$, as claimed.

We will refer to the characteristic $p$ condition in the above result as $F$ having characteristic big enough.

Lemma 4.2 Let $L$ be a Lie algebra over a field $F$. Consider the following two conditions:

(i) every $n$-maximal subalgebra of $L$ is contained in $N$; and

(ii) every $n$-maximal subalgebra of $L$ is a subideal of $L$. 
Then (i) implies (ii) and, if $F$ has characteristic zero or big enough, (ii) implies (i).

Proof. (i) $\Rightarrow$ (ii): It is clear that any subideal of $N$ is a subideal of $L$.

(ii) $\Rightarrow$ (i): Let $I$ be an $n$-maximal subalgebra of $L$ and suppose that it is a subideal of $L$. Then, under the extra hypothesis, it is a nilpotent subideal of $L$, by Lemma 2.2 and so is contained in $N$, by Lemma 4.1,

Clearly, if $L$ is solvable then a necessary condition for Lemma 4.2 (i) to hold is that $\operatorname{dim} L / N \leq n$, since there is a chain of subalgebras of length $n$ from $N$ to $L$. However, this condition is not sufficient, in general, as is clear from previous results and the following.

Theorem 4.3 Let $L$ be a supersolvable Lie algebra over a field $F$ of characteristic zero or big enough. Then every $n$-maximal subalgebra of $L$ is a subideal of $L$ if and only if either

(i) $L$ is nilpotent; or

(ii) $\operatorname{dim} L \leq n$.

Proof. Suppose that every $n$-maximal subalgebra of $L$ is a subideal of $L$, but that $L$ is not nilpotent, and let $N$ be the nilradical of $L$. Let

$$
0=A_{0}<A_{1}<\ldots<A_{k}=N<\ldots<A_{r}=L
$$

be a chief series for $L$ through $N$. Then each chief factor is one dimensional since $L$ is supersolvable and so $r=\operatorname{dim} L$. Let $x \in A_{r} \backslash A_{r-1}$. Then

$$
F x<A_{1}+F x<\ldots<A_{r-1}+F x=L
$$

is a maximal chain of subalgebras of $L$, and $F x$ is an $(r-1)$-maximal subalgebra of $L$. If $r>n$ it follows that $x$ belongs to an $n$-maximal subalgebra of $L$. Since $x \notin N$ this contradicts Lemma 4.2,

\section{References}

[1] D.W. Barnes, 'Nilpotency of Lie algebras', Math. Z. 79 (1962), 237238.

[2] D.W. Barnes, On the cohomology of soluble Lie algebras, Math. Z. 101 (1967), 343-349. 
[3] D.W. Barnes and H.M. Gastineau-Hills, 'On the theory of soluble Lie algebras', Math. Z. 106 (1968), 343-354.

[4] V. A. Belonogov, 'Finite solvable groups with nilpotent 2-maximal subgroups', Mathematical notes of the Academy of Sciences of the USSR 3 (1) (1968), 15-21.

[5] B. Huppert, 'Normalteiler und maximale Untergruppen endlicher Gruppen' Math. Z. 60 (1954), 409 - 434.

[6] D. V. Maksimenko, 'On action of outer derivations on nilpotent ideals of Lie algebras', Algebra Discrete Math. 2009, Issue 1, 74-82.

[7] A. Mann, 'Finite groups whose $n$-maximal subgroups are subnormal', Trans. Amer. Math. Soc. 132 (2) (1968), 395-409.

[8] E. Schenkman, 'A theory of subinvariant Lie algebras', Amer. J. Math. 73 (1951), 453-474.

[9] E.L. Stitzinger, 'A Note on a Class of Lie algebras', Math. Z. 116 (1970), $141-142$.

[10] H. Strade and R. Farnsteiner, 'Modular Lie algebras and their Representations', Marcel Dekker, New York and Basel (1988).

[11] D.A. Towers, 'A Frattini theory for algebras', Proc. London Math. Soc. (3) 27 (1973), 440-462.

[12] D.A. Towers 'Lie algebras all of whose proper subalgebras are nilpotent', Lin. Alg. Appl. 32 (1980), 61-73.

[13] D.A. Towers, 'C-Ideals of Lie algebras', Comm. Alg. 37 (2009), 4366 $-4373$.

[14] D.A. Towers, 'Subalgebras that cover or avoid chief factors of Lie algebras', arXiv:1311.7270v2 [math.RA] to appear in Proc. Amer. Math. Soc.

[15] V.R.VAREA, 'Lie algebras whose maximal subalgebras are modular', Proc. Roy. Soc. Edinburgh Sect. A 94 (1983), 9-13.

[16] V.R. VAREA, 'On modular subalgebras in Lie algebras of prime characteristic', Contemporary Math. 110 (1990), 289-307.

[17] V.R. VAREA, 'Lie algebras all of whose proper subalgebras are solvable', Comm. Alg. 23 (9), (1995), 3245-3267. 